\title{
ASPECTOS ECONÓMICOS Y ORGANIZACIONALES EN LA CREACIÓN DEL PARQUE TECNOLÓGICO DE LA REGIÓN DE KURSK
}

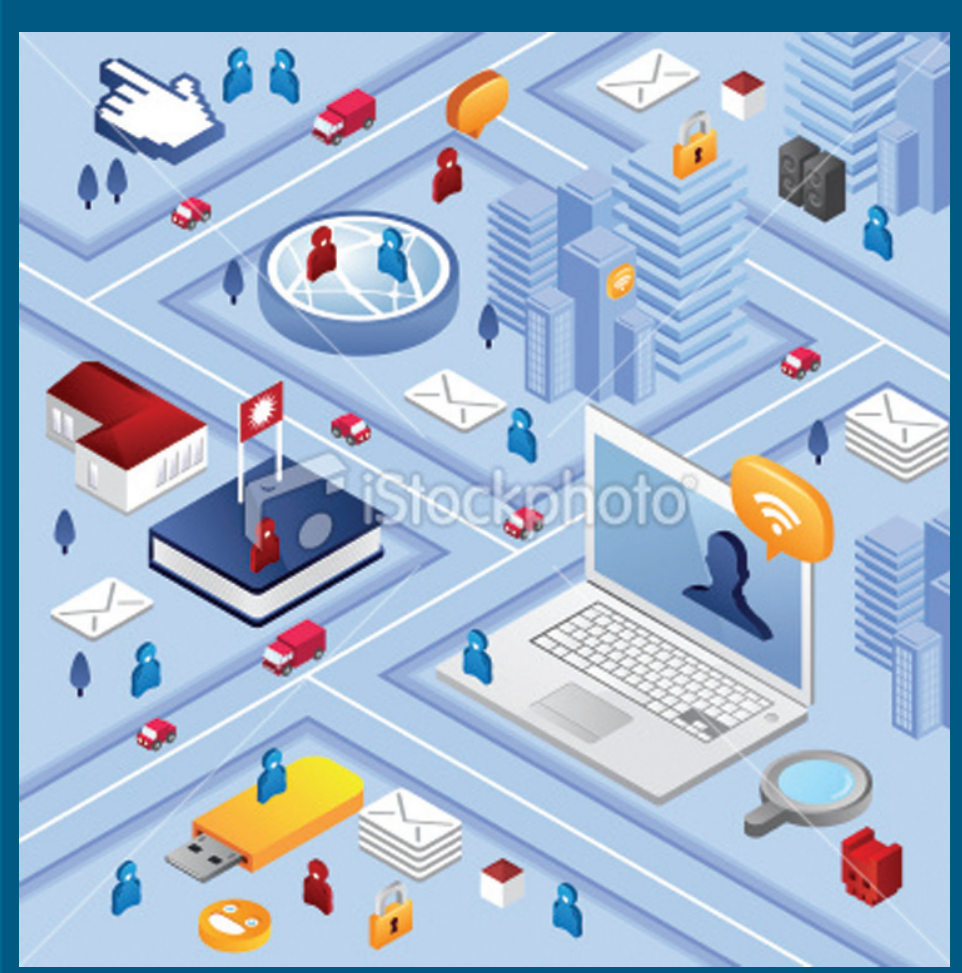

1 S.G. Emelyanov, L.N. Borisoglebskaya, A.A. Maltseva, Docentes de la Universidad Estatal Sur-Oeste, Federación de Rusia.

\section{S.G. Emelyanov, L.N. Borisoglebskaya, A.A. Maltseva,}

Resumen- En el artículo están analizados los aspectos de organización en la creación de Parques Tecnológicos en la Federación Rusa, se presenta una estructura funcional y organizativa del Parque Tecnológico de la región de Kursk. Con base en la sistematización de los materiales metódologicos, están definidas las direcciones de desarrollo del Parque Tecnológico, su objetivo y tareas, es examinada la lista de servicios de negocios propuestos y el estado ad hoc del Parque Tecnológico en el año 2014.

Abstract - In the article are discussed the questions of organization in development of technology parks in the Russian Federation, provides a functional and organizational structure of the Technological Park of the Kursk region. Based on the systematization of methodological materials are distinguished addresses Technology Park development, its aims and tasks, reviewed the list of business services offered and ad hoc status of the Technology Park in 2014.

Palabras Clave- parque tecnológico, plan de negocios, desarrollo de innovaciones, compañía de innovación, empresa gerencial, compañía de inversiones, incubadora de negocios, propiedad intelectual, transferencia de tecnologías, servicios de negocios.

Introducción

La creación de los Parques Tecnológicos por medio de la reforma de las zonas de producción es una de las direcciones básicas en la realización del escenario de innovación del desarrollo de Rusia en la etapa moderna. Con su realización se relaciona la decisión de los más importantes problemas sociales y económicos - el mejoramiento de la calidad de vida de los rusos y el aumento de la competitividad en la producción nacional. 
La creación de Parques Tecnológicos regionales se hace con el objetivo básico de suministrar el desarrollo acelerado en las ramas de alta tecnología y su transformación en una de las fuerzas motrices básicas del crecimiento económico del sujeto de la federación.

\section{Aspectos Organizativos en Creación de Parques Tecnológicos de la Región de Kursk}

La Universidad Técnica Estatal de Kursk elaboró el proyecto del Parque Técnico de la región de Kursk, que representara la estructura territorial y su distribución incluyendo el centro de producción innovador. En este centro se unen grandes compañías, pequeñas empresas de innovación, establecimientos de investigación científica y escuelas superiores que trabajan en direcciones de investigación y producción, las cuales se desarrollan tradicionalmente en la región de Kursk, tales como, construcción de maquinaria, construcción de aparatos de precisión, nanotecnologías, ITtecnologías.

\section{Durante}

realización del proyecto será creada la infraestructura que corresponde a los estándares internacionales, para esto se planea hacer la reconstrucción de cuerpos básicos del Parque Técnico, incluyendo locales para laboratorios y locales experimentales de producción, oficinas, centro para exposiciones y conferencias.

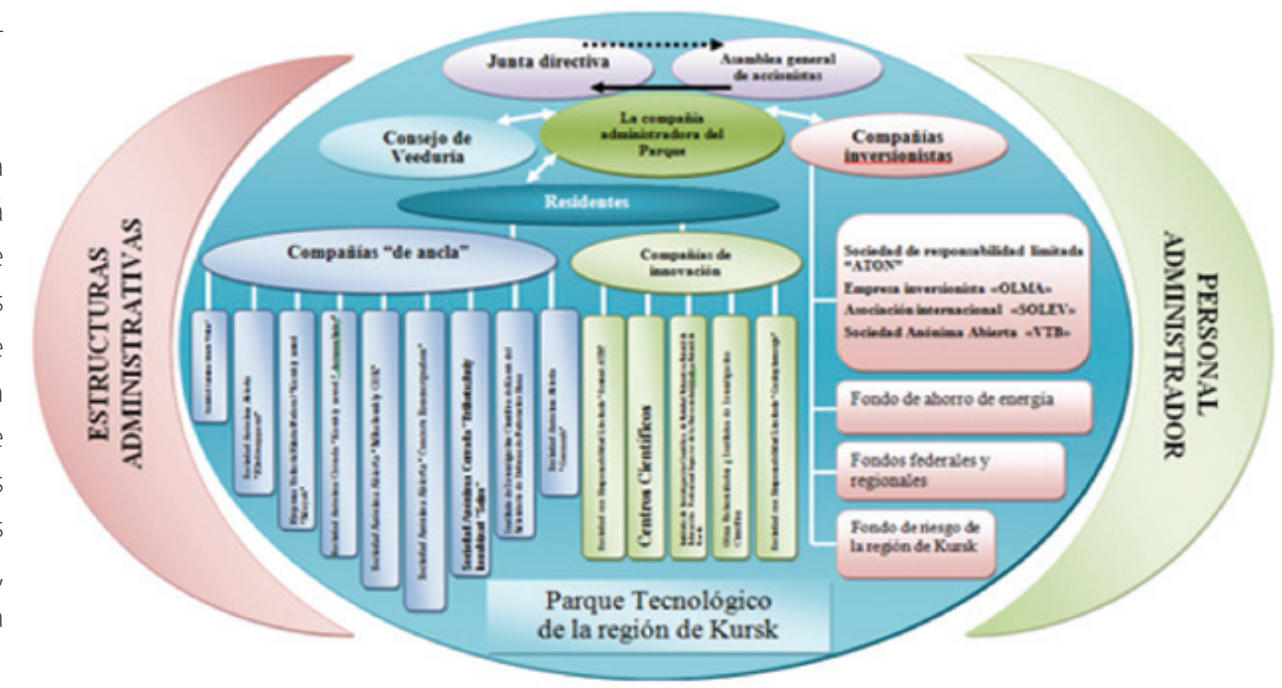

La compañía administradora del Parque Tecnológico es la compañía, que realiza el management del Parque Tecnológico, contribuye al desarrollo de negocios de los residentes, les presta servicios de negocios, dirige su posterior desarrollo.

Las compañías de servicios son organizaciones o subdivisiones estructurales de la empresa gerencial que prestan distintos tipos de servicios de oficina y otros servicios a los residentes del Parque Tecnológico.

La estructura administrativa del Parque Tecnológico de Kursk incluirá la compañía administrativa y las compañías "de ancla", de innovación y de inversiones (Fig. 1). La actividad de la compañía administrativa será regulada por la Junta directiva, elegida por la asamblea general de los accionistas, y será supervisada por el Consejo de Supervisión del Parque Tecnológico.
En el Parque

Tecnológico serán presentados todos los participantes del ciclo de innovación:

Las compañías "de ancla" son las grandes compañías a nivel regional, federal y mundial, cuya actividad es de perfil para el Parque Tecnológico.

Las compañías de innovación son las compañías medianas y pequeñas que cumplen con investigaciones fundamentales, trabajos de prospección y diseñó o que hacen la producción con la novedad científica demostrada.

Las compañías inversionistas son organizaciones de crédito e inversiones de formas varias: fondos de riesgo, bancos, fondos de inversiones directas, inversionistas particulares, otras estructuras que invierten medios financieros en la actividad de las compañías de innovación. 
forma administrativo-jurídica escogida. En la actualidad, para los objetivos de la creación del Parque Tecnológico, la forma más preferible es la sociedad anónima abierta, cuyo propietario será la administración de la región de Kursk.
Departamento de economía y finanzas responde por el control y preparación de informes de la compañía administradora del Parque Tecnológico, presta servicios de contabilidad a los clientes.

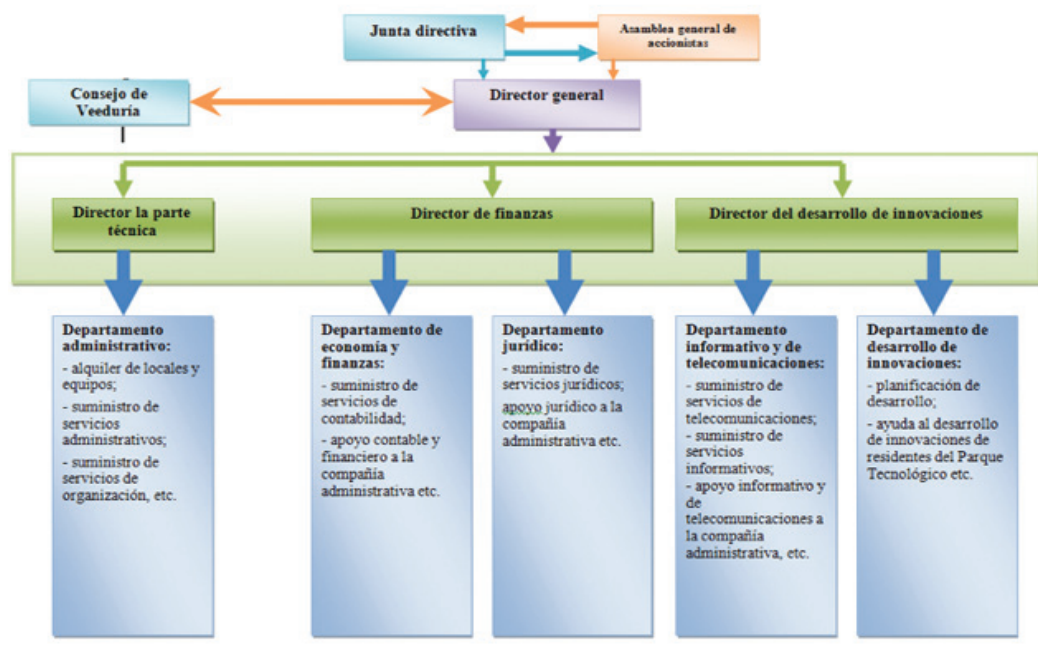

Figura 2. Estructura organizativa de la compañía administradora del Parque Tecnológico de la región de Kursk

La estructura funcional de la compañía administrativa del Parque Tecnológico de la región de Kursk se presenta en la Figura 3.

El departamento de desarrollo innovador elabora los planes estratégicos del trabajo del Parque Tecnológico, determina las nuevas direcciones de las actividades, realiza el análisis corriente del estado actual de los sectores, atrae a los residentes y los inversionistas.

El departamento administrativo responde por el mantenimiento de redes de ingeniería, edificios y locales del Parque Tecnológico, incubadoras de negocios, realiza su explotación técnica.

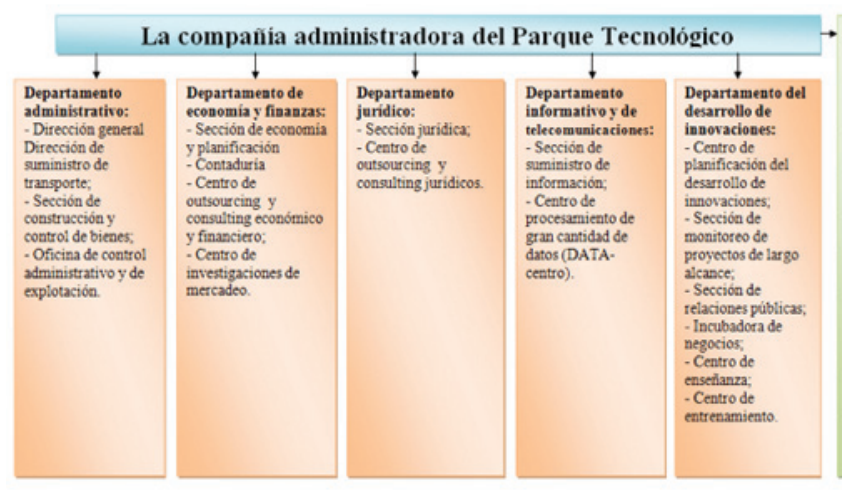

Figura 3. Estructura funcional del Parque Tecnológico de la región de Kursk

Departamento jurídico apoya jurídico a las actividades del Parque Tecnológico, presta a los residentes una serie de servicios y consultas jurídicas.
Departamento administrativo conjunto con el Departamento de economía y finanzas realiza la supervisión de la reconstrucción e introducción en la explotación de los locales del Parque Tecnológico

Departamento informativo y de telecomunicaciones presta apoyo informativo y de telecomunicaciones a la compañía administradora y a los residentes del Parque Tecnológico.

Infraestructura de innovaciones del Parque Tecnológico incluye las subdivisiones que prestan servicios especiales de negocios a los clientes residentes.

\section{Direcciones Básicas de la Actividad de la Compañía que Administra el Parque Tecnológico}

Basándose en el análisis de la práctica nacional y experiencia progresiva internacional en funcionamiento de Parques Tecnológicos, se destacaron las principales direcciones de actividades de la compañía que administra el parque Tecnológico de la región de Kursk:

- Control sobre el complejo de propiedad del PT cuyo objetivo es crear condiciones favorables para las actividades de las compañías-residentes;

- Garantía de incorporación de propiedad intelectual al movimiento administrativo y transformación de elaboraciones

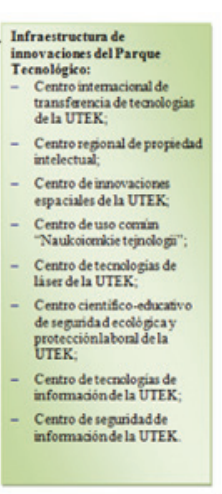
de innovación de las compañías regionales de gran capacidad científica a las industrias por fuera de la región de Kursk;

- Participación en la formación del sistemas de apoyo a pequeñas empresas de innovación por cuenta de prestación de servicios necesarios;

- Mejoramiento de las condiciones iniciales en la creación de negocios por medio de la concesión (durante 3 años) sobre la base contractual y las condiciones de privilegio a las empresas de innovación que forma parte del Parque Tecnológico, áreas de laboratorio y áreas experimentales de producción, equipo para la ejecución de trabajos de investigación científica asi como oficinas y lugares de exposición y conferencias; 
- Colaboración en la creación de pequeñas empresas innovadoras mediante prestaciones de todo tipo de servicios de consulta en la parte de ingeniería; ayuda en la inscripción de la pequeña empresa y colaboración jurídica en la certificación de productos;

- Concesión de servicios técnicos, tecnológicos e informativos, etc.;

- Consultas sobre la preparación de demandas de innovación y memorándumes de inversiones sobre imposición de impuestos de privilegio de los residentes del Parque Tecnológico;

- Investigación de mercados acerca de las actividades del Parque Tecnológico y prestación de servicios de mercadeo a empresas supervisadas;

- Busqueda de atracción inversionista para el desarrollo de negocios innovadores y creación de empresas de gran capacidad científica;

\section{- Control de empresas innovadoras;}

- Descubrimiento y selección de innovaciones de mercado efectivas y su posterior acompañamiento durante el ciclo innovador hasta su incorporación en la industria;

- Realización de contratos dentro de los limites de proyectos innovadores;

- Incorporación de los residentes del Parque Tecnológico en los programas regionales y federales;

- Soporte complejo de empresas-residentes en los mercados regionales, nacionales e internacionales.

\section{Objetivo y Tareas del Parque Tecnológico de la Región de Kursk}

En el proyecto del Parque Tecnológico de la región de Kursk, fue formulado el objetivo conceptual de su creación: formación de condiciones necesarias para el desarrollo innovador de la economía regional y uso efectivo del potencial científico-técnico, recursos materiales y financieros, que están dirigidos a la creación de tecnologías de gran capacidad científica y ahorro de recursos asi como también a la creación de unos nuevos tipos de productos para la región.

Las tareas del Parque Tecnológico, si hablamos mas concreto del objetivo de la creación, permiten destacar direcciones principales de su desarrollo:

- Creación de una estructura innovadora y científica, y condiciones para el desarrollo de pequeñas y medianas compañías innovadoras;

- Generación de investigaciones y elaboraciones científicas, acrecimiento de potencial innovador de la región, aceleración del desarrollo de empresas de alta tecnología en la

región;

- Desarrollo del Clúster (sistema que une diferentes tipos de organizaciones) técnico-informativo de gran capacidad científica en la región, en el cual el Parque Tecnológico será una unión entre pequeñas y grandes compañías, entidades de educación superior e institutos de investigación científica;

- Activación del desarrollo IT-sector (sector de tecnologías de información) en la región como resultado de funcionamiento de DATA-centro y en su base realización de IToutsorsing proyectos de las grandes empresas de la región de Kursk;

- Desarrollo de potencial profesional en ramas científico-técnicas, creación de nuevos puestos de trabajo para especialistas jóvenes y especialistas altamente calificados;

Medios para alcanzar los objetivos en la creación del Parque Tecnológico de la región de Kursk son:

1. Creación en el Parque Tecnológico de un acceso a los servicios de negocios mas actuales para la realización del potencial científico-técnico y comercialización de innovaciones en el territorio de la región: alquiler con condiciones favorables, uso de equipos de alta precisión, servicios de Data-centro, organización de exposiciones de innovaciones, "ferias de riesgo" (feria para representantes que quieren suministrar inversiones de riesgo), interacción con entidades educativas, suministro de todo tipo de consultas;

2. Suministro de la demanda solvente a las innovaciones por parte de compañías exitosas en el marco de la especialización en las ramas industriales del Parque Tecnológico, que es el resultado de la distribución e interacción de las principales compañías del Parque Tecnológico, las cuales trabajan en construcción de equipos, construcción de maquinas, nanotecnologías e IT-tecnologías.

\section{Complejo de Servicio de Negocios del Parque Tecnológico}

La tarea principal de la compañía administradora consiste en suministrar a los residentes la totalidad del complejo de servicios, usando toda la cadena de creación del valor agregado, desde la estadía de NIOKR (actividades de investigación científica, experimental y de construcción) hasta la determinación de flujos logísticos de la producción final del residente. 
La estrategia competitiva de suministro de servicios de negocios en el Parque Tecnológico se muestra en la Figura 4.

traslado de residentes; limpieza de locales y territorio, adecuación del entorno; servicio público (infraestructura de acueducto y electricidad, canalización y otros), suministro de vigilancia y otros;

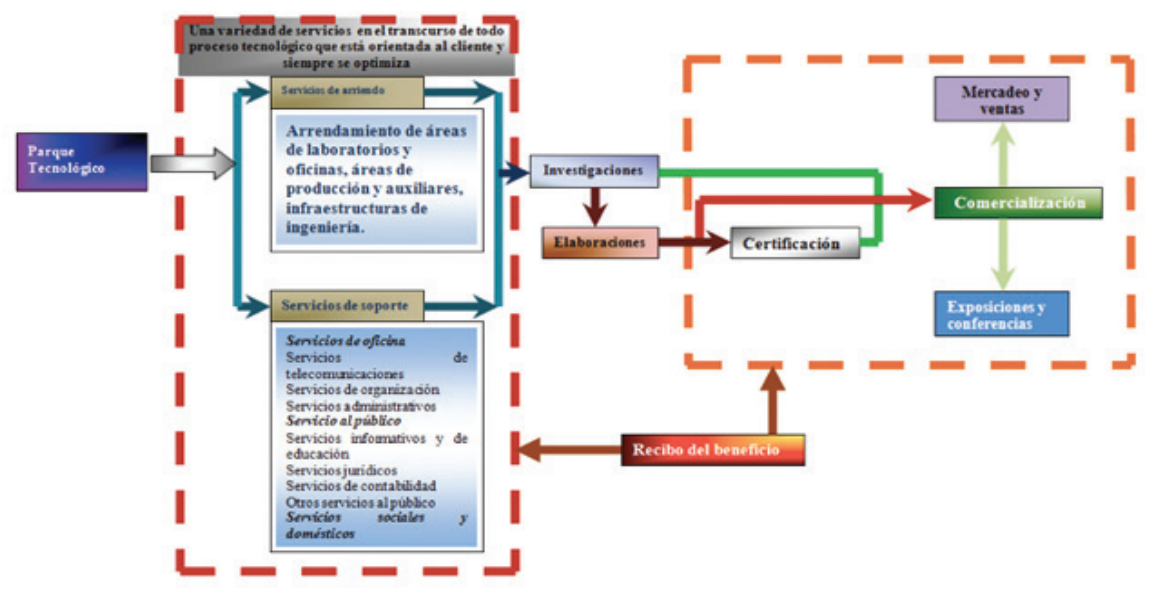

Suministro de recursos y logística: suministro de energía, abastecimiento centralizado con materia prima, servicios de bodega, servicios de transporte, organización de traslados por tren y realización de producción.

\section{Servicio al público}

De información y de educación

- Recolección, sistematización y análisis de información

Figura 4 - Estrategia competitiva del Parque Tecnológico

En el futuro a los residentes del Parque tecnológico les será prestado un complejo de servicios, que abarca las siguientes direcciones.

\section{Servicios de alquiler de inmuebles}

Arrendamiento con facilidad de pago a los residentes del Parque Tecnológico de áreas de laboratorios y oficinas, áreas de producción y auxiliares, infraestructuras de ingeniería.

\section{Servicios de oficina}

Servicios de telecomunicaciones

Servicios de comunicaciones;

Servicios de DATA-center:

- Conservación de grandes volúmenes de datos; - Creación de archivos estructurados de diversa información (texto,grafica,cartográfica, modelosen3D,esquemasyplanos vectoriales, multimedia) con la posibilidad de selección libre; - Modelación de dispositivos y mecanismos complejos, de procesos tecnológicos para las necesidades de la industria; - Ejecución de cálculos de grandes recursos en los intereses del cliente.

\section{Servicios de organización}

$$
\text { Organización }
$$

de

exposiciones, seminarios,

conferencias,

las instalaciones del centro de conferencias

\section{Servicios de administración}

Manejo de inmuebles y trabajos de construcción: arreglo y reparación de edificios y locales; ubicación y con la temática del Parque Tecnológico; - Consultas, dirigidas a la recepción de nuevos conocimientos en el campo de innovación y administración; - Elaboración de estrategias de mercadeo; - Elaboración de estrategias de propaganda de participantes de la actividad innovadora; - Realización investigativa de mercadeo y análisis de mercado de productos de innovación; - Proyección al mercado de productos (trabajos, servicios) para sectores económicos de alta tecnología; - Análisis de efectividad en actividades de mercadeo de integrantes de las actividades de innovación;

Servicios jurídicos

- Acompañamiento jurídico complejo de las actividades de innovación y consultas jurídicas;

\section{Servicios de contabilidad}

- Teneduría de libros, rendición del estado de cuentas en condiciones de outsoursing, consultas sobre preguntas indicadas.

\section{Otros servicios al público}

- Consultas de negocios en acompañamiento de proyectos; - Búsqueda de inversionistas para realización de proyectos; - Realización de cooperación activa en realización de tecnologías, productos y servicios de empresas y organizaciones por fuera de la región con base en centro Internacional de transferencia de tecnologías;

- Colaboración a los actores de la actividad innovadora en la preparación calificada de materiales para proteger su propiedad intelectual;

- Peritación, valorización, vigilancia jurídica y realización comercial de objetos de propiedad intelectual;

- Suministro de servicios de expertos en valorización, que se necesitan en el proceso de comercialización de resultados 
de actividades científico-técnicas;

- Ayuda a organizaciones para recibir la documentación necesaria: licencias, acuerdos, certificados.

- Sociales y domésticos.

- Organización de alimentación y otros.

\section{Conclusión}

Dentro de la realización de proyecto se propone

el siguiente estado del Parque Tecnológico hasta el 2014:

- Terminación de reconstrucción de redes de ingeniería y objetos de infraestructura de ingeniería;

- Entrega en alquiler de locales industriales y de oficinas a los recidentes para explotación;

- Creación del mecanismo de búsqueda de proyectos y tecnologías de innovación;

- Formación del mecanismo de incorporación de tecnologías de innovación;

- Organización del sistema de inversiones en innovaciones;

- Política activa de mercadeo en la parte de atracción de nuevos residentes para su instalación en el territorio del Parque tecnológico;

- Creación del sistema de preparación de personal para la economía innovadora;

- Formación del medio innovador en la región de Kursk y fortalecimiento de su rol a nivel regional;

- Crecimiento de ritmos de desarrollo del sector industrial en la región, desarrollo de ramas consumidoras de productos y servicios del Parque Tecnológico, crecimiento del producto bruto regional, crecimiento de las ganancias del presupuesto por cuenta del incremento a los impuestos;

- Creación de nuevos puestos de trabajo y crecimiento del nivel de vida de la población, la conservación y la atracción en la región de los especialistas altamente calificados.

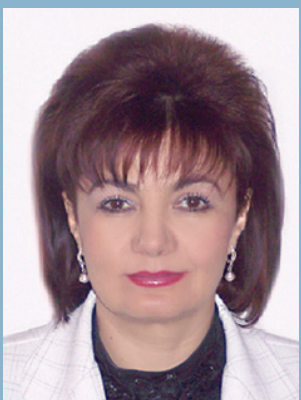

Larissa Nikolaevna Borisoglebskaya - Doctor en ciencias económicas, Professor, Pro-rector de Ciencias de la Universidad Técnica Estatal de Kursk.

En 2004 obtiene el grado de Grand Ph.D y en el año 2005 le otorgan el título del Professor.

En 2006 Decano de la Facultad de Negocios y Servicios, de la Universidad Estatal de Belgorod, y Director de la Cátedra Economía y Control de Empresas.

Año 2007, sud-director de proyecto regional "Estrategia de desarrollo social y económico de la Región de Kursk hasta el año 2020".

Año 2007, Director del proyecto del Fondo Científico Humanitario Ruso "Estudio del potencial de innovación de la región de Belgorod con la finalidad de creación y desarrollo del centro de transferencia de tecnologías".

Año 2007, Director del proyecto auspiciado por la Universidad Estatal de Belgorod "Marco Metodológico para la creación y la administración del centro la transferencia de las tecnologías en base a la formación del potencial de innovación de la Región".

Año 2008, sub-director de proyecto regional "Estrategia de desarrollo social y económico de la Región de Belgorod hasta el año 2025".

Año, 2008, Ejecutor responsable del proyecto auspiciado por la Universidad Técnica Estatal de Kursk: "Estrategia de desarrollo innovador de la Universidad Técnica Estatal de Kursk".

Fue miembro Consejo Público de la Gobernación de la región de Kursk, y del Consejo Económico del Gobierno de la región de Kursk, ayudante de diputado de la Duma del Estado Ruso. En su campo de interés científico están: las cuestiones estratégicas para el incremento de la eficacia y capacidad competitiva de la economía Rusa y Regional; la formación de los mecanismos económicos para la gerencia del Estado y de Organizaciones Municipales; la mejora y la creación de sistemas integrados de gerencia; mejoramiento del negocio de las empresas de innovación y control mediante procesos de innovación e inversión; investigación de los procesos sociales y económicos 